\title{
Critères de choix des matériaux et techniques d'empreinte en Prothèse Amovible Partielle
}

\section{RÉSUMÉ}

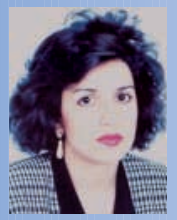

Nadia MERZOUK

Professeur d'Enseignement supérieur, Service de Prothèse Adjointe,

Université Mohamed V,

Faculté de médecine dentaire de Rabat, BP 6212, Rabat-Instituts, Maroc.

Salwa BERRADA

Professeur d'Enseignement Supérieur, Service de Prothèse Adjointe

Université Mohamed $V_{\text {, }}$

Faculté de médecine dentaire de Rabat, BP 6212, Rabat-Instituts, Maroc

\section{Faiza BENFDIL}

Professeur assistant,

Service de Prothèse Adjointe,

Université Mohamed V,

Faculté de médecine dentaire de Rabat,

BP 6212, Rabat-Instituts, Maroc

\section{Ahmed ABDEDINE}

Professeur d'Enseignement Supérieure Chef du service de Prothèse Adjointe, Université Mohamed V,

Faculté de médecine dentaire de Rabat BP 6212, Rabat-Instituts, Maroc.

L'empreinte constitue une étape capitale de la réalisation d'une PAP. Elle participe à la recherche de l'équilibre prothétique et doit tenir compte des comportements physiologiques différents des structures d'appui. La diversité des situations cliniques à l'origine d'une variété des techniques d'empreinte et la multiplicité des matériaux de plus en plus performants, exposent le praticien à une situation de confusion lors du choix des matériaux et techniques d'empreinte. Après l'étude des principales propriétés des matériaux permettant de mieux comprendre leurs limites et une brève description des techniques d'empreinte, nous proposons une analyse des éventuels facteurs cliniques et techniques ayant une incidence sur le choix des matériaux et des techniques d'empreinte. Cet essai de simplification réfléchi et raisonné, permet d'optimiser l'empreinte et de conduire avec succès la thérapeutique prothétique.

- dualité tissulaire

- matériaux à empreinte

- empreinte

- facteurs de choix des matériaux à empreinte
AOS 2008:243:265-278

DOI:10.1051/AOS:2008034

(C) AEOS / EDP Sciences 


\section{Introduction}

$>$

Les difficultés rencontrées au quotidien par de nombreux praticiens lors de la réalisation d'une Prothèse Amovible Partielle (PAP) relèvent de trois caractères spécifiques de ce type de prothèse : I'amovibilité, la mixité de l'appui et la diversité des situations cliniques. Pour vaincre ces difficultés, nous sommes amenés à assurer ce que l'on a coutume de nommer l'équilibre prothétique. La première approche de l'équilibre entre la prothèse et son environnement commence désormais dès le stade des empreintes, par la recherche des facteurs classiques de la triade d'équilibre de Housset: sustentation (vocation de l'empreinte primaire), rétention (vocation de l'empreinte secondaire) et stabilisation (vocation de l'empreinte tertiaire). Avant de présenter les différentes techniques d'empreinte spécifiques à la PAP et les principaux matériaux utilisés dans ce domaine, nous consacrerons un chapitre préliminaire à quelques notions fondamentales.

\section{Notions fondamentales}

\section{Comportement}

des surfaces d'appui

à l'égard d'une PAP

La PAP est une prothèse à appui mixte : elle s'appuie sur deux structures de nature et de comportement biomécanique différents.

Si une pression est exercée au niveau des surfaces d'appui dento-parodontales, la compression desmodontale permet à la dent un enfoncement dans son alvéole d'une amplitude d'environ 1/10 $\mathrm{mm}$ pour un parodonte sain ; la même pression exercée au niveau des structures ostéo-muqueuses entraîne une dépression muqueuse d'une amplitude de 0,4 à $1 \mathrm{~mm}$ (fig. 1)[1]. La déformation muqueuse est nettement plus importante que celle du desmodonte mais la récupération de ce dernier est totale et plus rapide[2].

L'analyse du diagramme ci-dessous représentant les résultats des recherches réalisées par Kydd et Dally sur les modifications des muqueuses buccales lors de la compres- sion provoquée par des éléments prothétiques (fig. 2), permet de déduire que :

- la muqueuse, sous l'effet de la prothèse, passe par une phase de déformation qui se décompose en une première phase élastique (la muqueuse perd de $100 \%$ à $50 \%$ d'épaisseur), suivie d'une phase visqueuse (la muqueuse perd de $5 \%$ à $45 \%$ de son épaisseur). Pendant cette phase visqueuse de

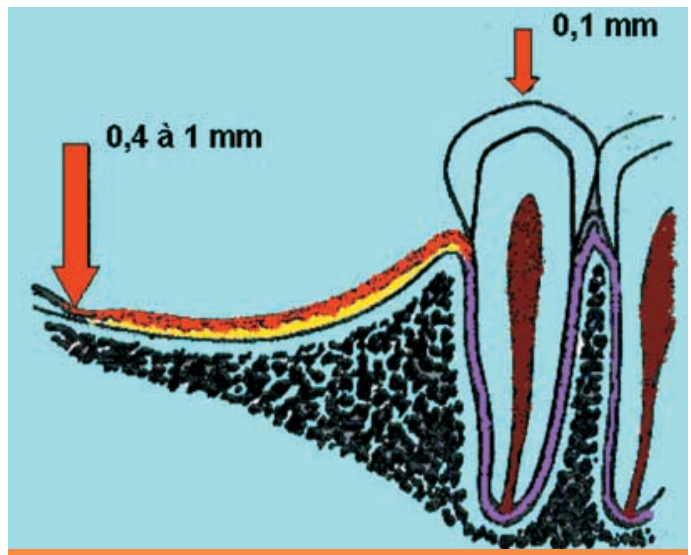

Fig. 1 Notion de dualité tissulaire[1] 


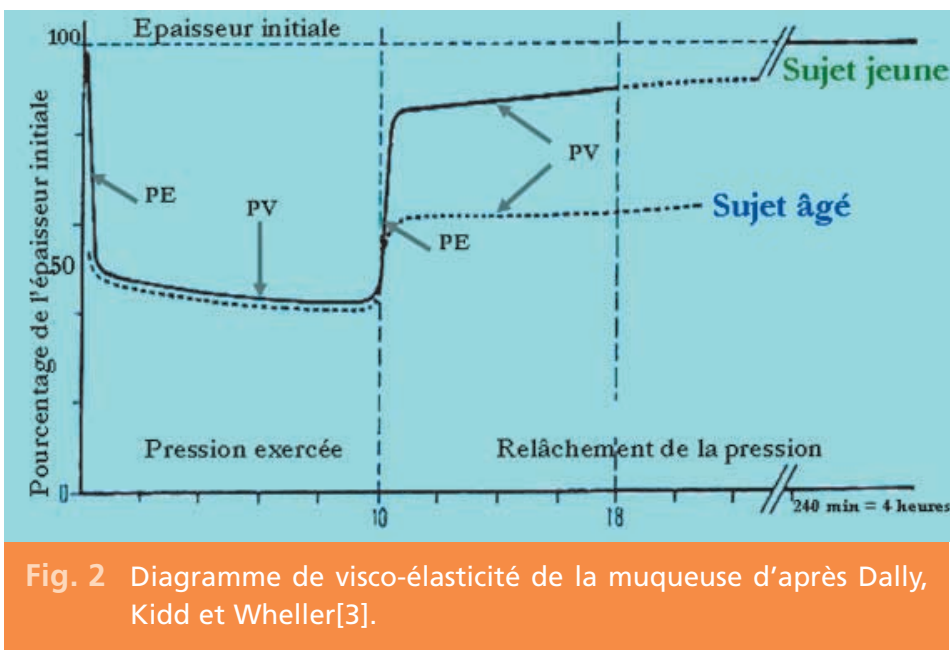

déformation, des cellules sanguines et lymphatiques sont rapidement chassées[3] ;

Quand la pression cesse, le phénomène est identique mais se produit à l'inverse ; la phase de récupération elle-même se décompose en une phase élastique de récupération rapide (la muqueuse passe de $45 \%$ à $70 \%$ d'épaisseur) suivie d'une phase visqueuse de récupération lente (la muqueuse passe de $70 \%$ à $90 \%$ d'épaisseur) ; les cellules sanguines et lymphatiques réhabitent lentement leur site initial : c'est le phénomène de visco-élasticité. De plus, si le seuil de viscoélasticité est dépassé, la fibromuqueuse sera écrasée, vidée de son eau et on assistera à des dégénérescences structurales [4]. - Si l'appui mixte de la prothèse amovible partielle est à l'origine d'une différence de dépressibilité tissulaire, il en est de même pour ce qui est de son appui qualitatif au sein des structures ostéomuqueuses qui n'offrent pas la même valeur de sustentation :

- les crêtes édentées et le palais recouverts de fibromuqueuse ferme et adhérente à l'os sous jacent sont plus favorables à la sustentation, donc à exploiter :

- les zones incompressibles situées au niveau du raphé médian, du sommet des crêtes et de la ligne oblique interne sont à décharger ;
- les insertions freinales et ligamentaires, trop dépressibles sont à éviter ;

- les zones avec fibromuqueuse épaisse, dépressible, recouvrant du tissu sous muqueux au niveau de la ligne de réflexion muqueuse et des zones de Schröeder, offrent une meilleure sustentation si leur compression ne dépasse pas le seuil de tolérance.

En terme de conclusion à ce premier volet, nous retenons cinq points essentiels pour un enregistrement correct des surfaces d'appui en PAP :

1) Préparation des surfaces d'appui : préalable indispensable pour diminuer toute compression desmodontale et toute déformation muqueuse exagérées ;

2) Repos tissulaire: nécessité absolue pour tout porteur de prothèse amovible partielle à prédominance d'appui ostéomuqueux ;

3) Anticipation du phénomène de dualité tissulaire : facteur déterminant de la finalité des empreintes en PAP ;

4) Recherche de la sustentation qualitative : condition nécessaire pour une meilleure exploitation des structures ostéomuqueuses ;

5) Compression dosée et mesurée : seul garant du respect de la fibromuqueuse. 


\section{Facteurs physiques de rétention}

La rétention prothétique est le facteur dont le défaut est le plus souvent mis en cause et représente une source fréquente de doléances. Il constitue ainsi, un objectif pour assurer le succès de l'équilibre prothétique.

En PAP, la rétention dépend de facteurs mécaniques, anatomiques et physiques.

Les lois physiques prennent le dessus chaque fois que l'édentement partiel est à prédominance d'appui ostéomuqueux. La recherche d'une rétention optimale, à défaut d'éléments mécaniques, conduit logiquement à enregistrer un maximum de surfaces d'appui ostéomuqueuses avec un film salivaire le plus fin et le plus étendu possible sur toute la surface de I'intrados, en particulier au niveau des bords et d'une partie de l'extrados[5].

C'est dans cette optique que les bords devront être les plus larges et les plus hauts possibles sans surextensions ni surépaisseurs, assurant une meilleure herméticité prothétique au moyen d'un joint périphérique sur des tissus mous et dépressibles (fig. 3) [6].

Pour atteindre ces objectifs, il est nécessaire de passer par deux empreintes complémentaires

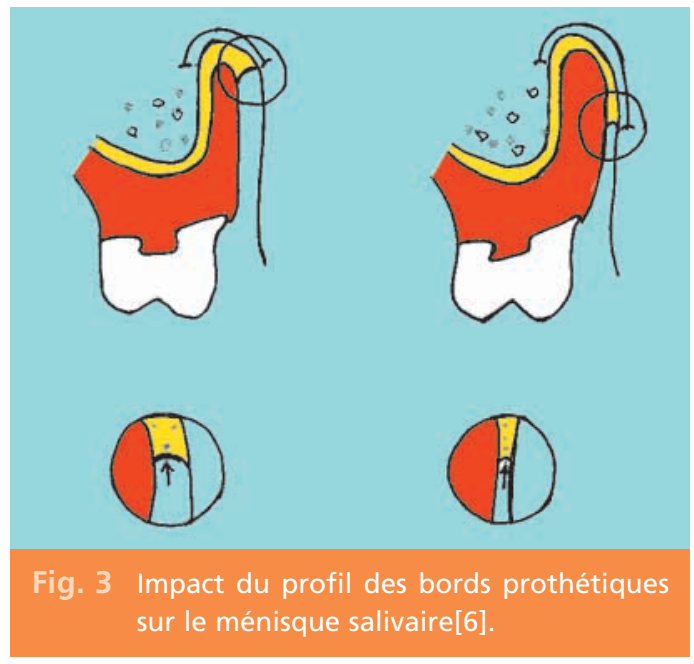

lors de l'enregistrement des surfaces d'appui à prédominance ostéomuqueuse : primaire à la recherche de la sustentation quantitative (surfaces d'appui maximales) et qualitative (surfaces favorables) et secondaire à la recherche de la rétention par adhésion (rôle du joint périphérique)[7].

Par ailleurs, l'utilisation d'un porte-empreinte individuel, le rinçage et l'assèchement buccal sont autant de paramètres qui permettent d'assurer une intimité et un ajustement le plus proche possible entre la prothèse et ses structures propres, procurant ainsi un meilleur confort au patient[8].

\section{Les matériaux d'empreinte}

Les matériaux d'empreinte sont nombreux et variés. Chacun présente des caractéristiques qui le différencient des autres matériaux.

En PAP, les matériaux à empreinte doivent répondre aux exigences des enregistrements pour Prothèse Fixée et Prothèse Amovible Complète.

Ainsi les qualités requises de ces matériaux sont les suivantes[9] :
- compatibilité avec les tissus et les matériaux de réplique ;

- fidélité :

- précision ;

- viscosités rapprochées ;

- temps de travail et de prise suffisants ;

- plasticité avant la prise et élasticité après la prise ; 
Tableau I

Comparaison des principales propriétés des matériaux à empreinte.

\begin{tabular}{|c|c|c|c|c|c|c|}
\hline & $\begin{array}{l}\text { Temps de } \\
\text { prise }\end{array}$ & $\begin{array}{l}\text { Hydro- } \\
\text { philie }\end{array}$ & Fidélité & Élasticité & $\begin{array}{c}\text { Stabilité } \\
\text { dimensionnelle }\end{array}$ & Coulée \\
\hline Alginate & 3 à $4 \mathrm{mn}$ & ++ & ++ & + & + & $15 \mathrm{mn}$ \\
\hline Pâte Zno & 6 à $7 \mathrm{mn}$ & +++ & ++ & - & +++ & $24 \mathrm{~h}$ \\
\hline Polysulfures & 8 à $12 \mathrm{mn}$ & - & ++ & ++ & ++ & $1 \mathrm{~h}$ \\
\hline $\begin{array}{l}\text { Silicones par } \\
\text { condensation }\end{array}$ & 4 à $5 \mathrm{mn}$ & - & + & ++ & ++ & $15 \mathrm{mn}$ \\
\hline $\begin{array}{c}\text { Silicones } \\
\text { par addition }\end{array}$ & 3 à $4 \mathrm{mn}$ & ++ & +++ & ++ & +++ & $12 \mathrm{~h}$ \\
\hline Polyéthers & 4 à $5 \mathrm{mn}$ & +++ & +++ & +++ & +++ & $24 \mathrm{~h}$ \\
\hline
\end{tabular}

- prix de revient en accord avec les résultats souhaités.

Pour déterminer le matériau qui répond le mieux aux critères de qualité que nous venons d'énoncer, nous nous proposons de présenter à travers le tableau suivant, une étude comparative des principales propriétés des matériaux d'empreinte (tableau I).

Il ressort de cette étude comparative que tous les matériaux sont compatibles et que, malgré les avantages des uns par rapport aux autres, on ne peut discriminer aucun matériau.

Sur le plan précision d'enregistrement, il faut distinguer entre la précision dimensionnelle qui intéresse la globalité de l'empreinte et la fidélité qui désigne la capacité d'enregistrement des détails[10-11].

Tous les matériaux présentent des précisions dimensionnelles suffisantes mais des propriétés de fidélité différentes.

Les polyéthers, en raison de la fluidité qu'ils présentent à l'injection et de la rigidité qu'ils ont après la prise, permettent l'enregistrement des plus petits détails (rainures et cannelures des attachements). Ce sont les plus fidèles suivis des silicones par addition.

La fidélité est influencée par des facteurs tels que : la mouillabilité, la viscosité et le caractère hydrophile du matériau à empreinte.

Un matériau de faible viscosité mouillera plus rapidement les surfaces dentaires qu'un matériau de plus haute viscosité[9].

A viscosité égale, la mouillabilité des polyéthers est meilleure que celle des silicones[12]. Par ordre de mouillabilité croissante, on peut classer les silicones hydrophobes, les polysulfures, les silicones hydrophiles et les polyéthers[9].

Les pâtes à l'oxyde de zinc eugénol (ZNO) mouillent parfaitement les surfaces muqueuses en raison du caractère hydrophile de l'eugénate de zinc[7].

L'hydrophilie est une condition nécessaire à la mouillabilité, cette qualité est l'apanage 
de plusieurs matériaux mais pas des polysulfures et des silicones dits «hydrophobes»[13].

Les silicones modernes «hydrophiles» ou «hydroactifs» ont vu leur mouillabilité s'améliorer par l'adjonction d'agents tensioactifs ou surfactants [14].

Les polysulfures les plus hydrophobes ne permettent pas d'obtenir des empreintes très détaillées. Les surfaces lisses, voire glacées, obtenues à l'aide de ce matériau, présentent un intérêt en prothèse amovible dans la mesure où elles facilitent le mouillage des intrados par la salive [7, 15].

Les polyéthers présentent une résistance au déchirement et à la déformation légèrement supérieure à celle des silicones et des polysulfures. Les alginates résistent moins au déchirement.

Les pâtes à l'oxyde de zinc, en raison de leur caractère inélastique, ne peuvent passer les contres dépouilles.

La stabilité dimensionnelle très élevée des polyéthers permet de différer le traitement de l'empreinte; il a été même montré que I'on peut couler 2 à 3 fois une empreinte aux polyéthers sans perte de précision[9].

Les silicones par addition et les pâtes à l'oxyde de zinc ont une stabilité dimensionnelle importante[9,16].

La faible stabilité dimensionnelle des polysulfures (liée au phénomène de réticulation prolongé), des silicones de première génération (liée au phénomène de réticulation par condensation) et des alginates (liée aux phénomènes de synérèse et d'imbibition) impose de les traiter sans tarder après la prise de l'empreinte[9, 16, 17].

L'enregistrement de la limite d'action muscu-

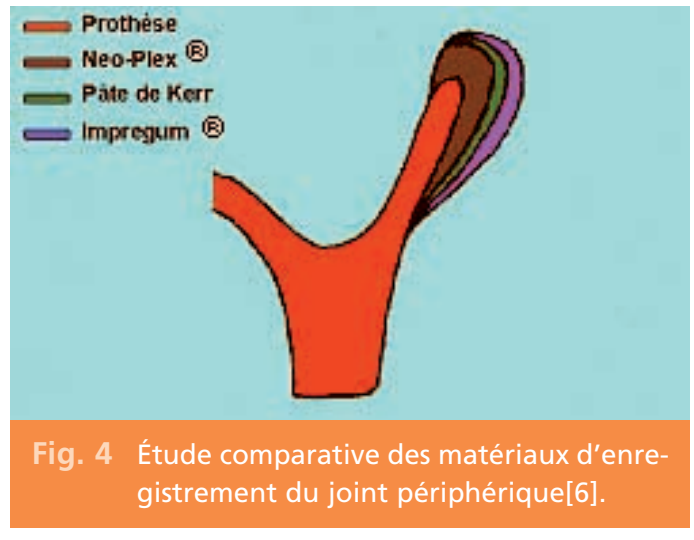

laire nécessite des temps de travail et de prise suffisants.

Les polysulfures et la pâte de Kerr laissent amplement le temps au praticien d'enregistrer correctement les mouvements des organes périphériques.

Les polyéthers et les silicones, modernes par I'allongement de leur temps de prise, permettent de réaliser des joints périphériques «en toute sérénité» quand la pâte de Kerr ne peut être employée (contre dépouille)[6, 18].

Le temps de prise des silicones, légèrement inférieur à celui des polyéthers, joue un intérêt considérable dans certaines situations cliniques[19].

Les joints périphériques aux élastomères peuvent être réalisés secteur par secteur ou en globalité.

Une étude comparative de trois matériaux d'enregistrement des joints périphériques (fig. 4) réalisée par Naser et coll[6] permet de conclure que c'est I'Impregum ${ }^{\circledR}$ (polyéther) qui permet d'obtenir les bords les plus épais et les plus hauts, à l'inverse du Néoplex ${ }^{\circledR}$ (polysulfure). La pâte de Kerr donne des résultats intermédiaires entre ces deux matériaux. 


\section{Les empreintes en PAP}

L'empreinte en PAP vise les objectifs suivants :

- reproduire des éléments anatomiques très différents dans leur structure et leur rôle ;

- tenir compte de la différence de dépressibilité tissulaire pour que la prothèse soit plaquée sur ses structures avec une légère pression au niveau muqueux ;

- enregistrer le jeu des organes périphériques pour exploiter au mieux les zones qui permettent de stabiliser la prothèse.

La répartition des forces fonctionnelles sur les éléments dentaires et sur le support ostéomuqueux confère aux empreintes deux expressions cliniques selon le degré et la localisation de l'édentement.

\section{Empreintes globales}

Elles enregistrent en même temps l'ensemble de I'arcade : structures dento-parodontales et ostéomuqueuses.

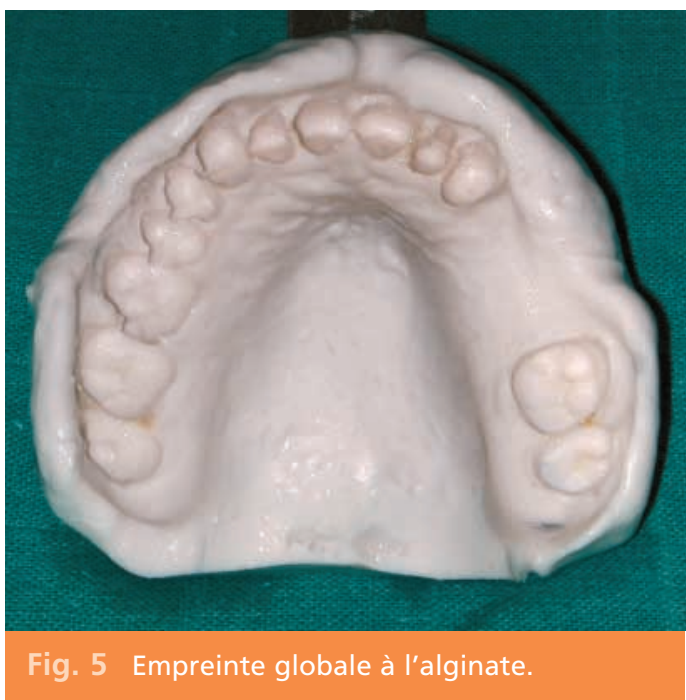

Ces empreintes sont destinées aux édentements intercalés ( $\mathrm{Cl}$ III et CI IV de KennedyApplegate) où le nombre et la valeur des dents absentes sont inférieurs à ceux des dents restantes. Elles font appel le plus souvent à des porte-empreintes standards et aux hydrocolloïdes irréversibles (Alginate $\mathrm{Cl}$ A) (fig. 5).

Dans des situations complexes, il est indiqué parfois d'avoir recours à un porte-empreinte individuel et aux élastomères en une seule viscosité ou en double viscosité comme la technique du double mélange utilisée en prothèse fixée (fig. 6).

\section{Empreintes composées}

Ces empreintes présentent la particularité de dissocier l'enregistrement des structures dento-parodontales de celui des structures ostéomuqueuses quand l'appui muco-osseux est prédominant et ce dans l'esprit d'une meilleure anticipation du phénomène de dualité tissulaire. Les empreintes composées peuvent être globales ou partielles.

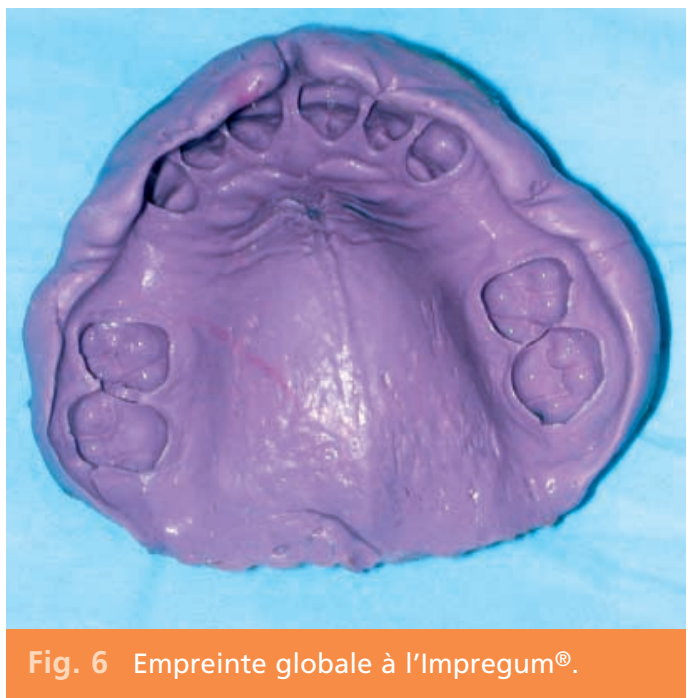




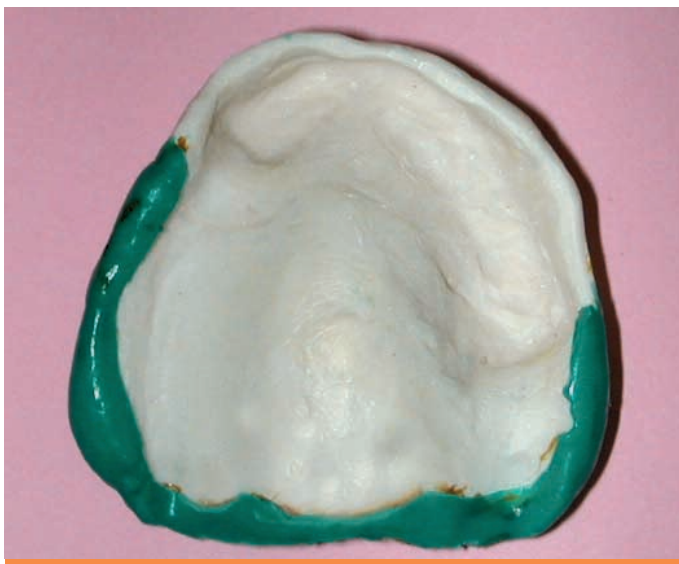

Fig. 7a Joint périphérique au Xantopren vert ${ }^{\circledR}$.

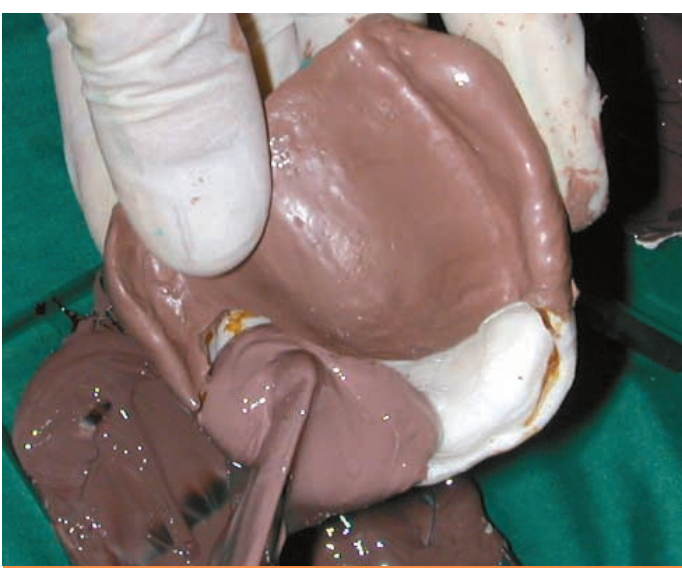

Fig. 7c Garnissage du P.E.I en regard des dents avec Permlastic Regular ${ }^{\circledR}$.

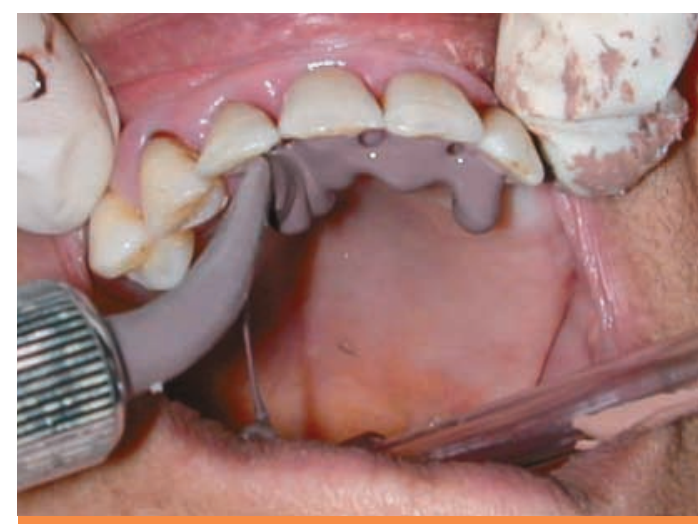

Fig. 7e Injection du Permlastic Light ${ }^{\circledR}$ au niveau dentaire.

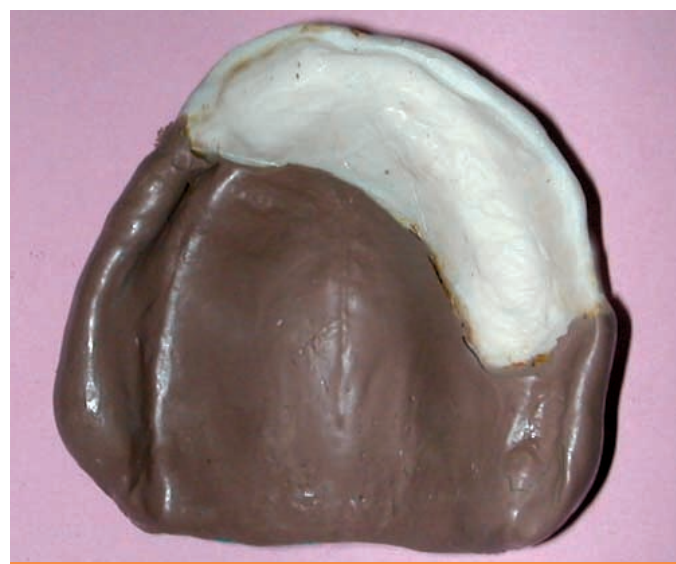

Fig. 7b Empreinte de stabilisation au Permlastic Regular ${ }^{\circledR}$

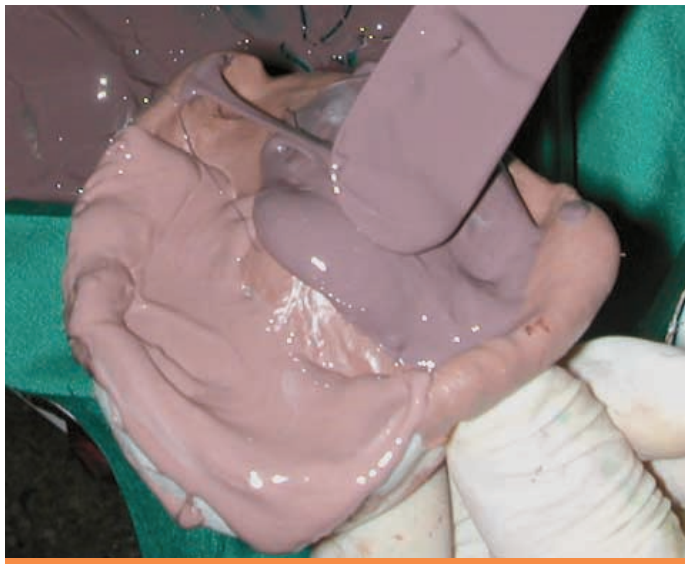

Fig. 7d Lavis de l'empreinte de stabilisation avec Permlastic Light ${ }^{\circledR}$.

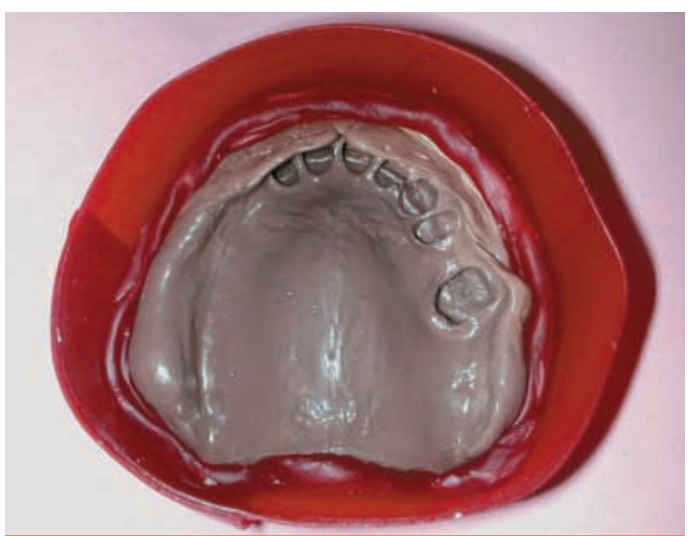

Fig. $7 f$ Empreinte finale coffrée.

Fig. 7 a à $f$ Empreinte composée globale. 


\section{$>$ Empreintes composées globales}

Elles sont indiquées dans les édentements encastrés de grande étendue ( $\mathrm{Cl}$ IV et $\mathrm{Cl} V$ de Kennedy-Applegate) et dans les édentements terminaux maxillaires ( $\mathrm{Cl}$ I et $\mathrm{Cl}$ II de KennedyApplegate).

L'empreinte composée globale est une empreinte anatomo-fonctionnelle qui fait appel à un porte-empreinte individuel et aux matériaux de viscosités rapprochées. La technique repose sur le même principe de la «washtechnic» employée en prothèse fixée.

Après correction du porte-empreinte individuel, l'enregistrement de la limite d'action musculaire est réalisé secteur par secteur au moyen de la pâte de Kerr ou en globalité au moyen des élastomères (silicones et polyéthers) (fig. 7a).

L'enregistrement des structures d'appui se décompose en deux temps; le premier consiste à enregistrer les surfaces ostéomuqueuses au moyen d'un matériau de moyenne viscosité, le second consiste en un enregistrement de la totalité de l'arcade au moyen de deux viscosités (fig. 7b à 7e).

Afin de préserver le joint périphérique, un coffrage de l'empreinte est réalisé comme en Prothèse Amovible Complète (fig. 7f).

\section{> Empreintes composées partielles}

Ces empreintes sont réservées aux édentements de $\mathrm{Cl}$ I et $\mathrm{Cl}$ II de Kennedy-Applegate mandibulaires de moyenne étendue[20].

Les surfaces dento-parodontales sont enregistrées en premier au moyen de l'alginate et du porte-empreinte standard.

Le châssis réalisé à partir de ce premier enregistrement, est muni de selles porte-empeintes pour enregistrer les surfaces ostéomuqueuses (fig. $\mathbf{8 a}$ et $\mathbf{8 b}$ ). Le modèle de travail est ensuite corrigé (fig. 8c).

Dans le cas d'inflammation tissulaire, le châssis est muni de selles en résine qui vont véhiculer la résine à prise retard pour assurer la remise en état des tissus : c'est l'empreinte ambulatoire (fig. 9).
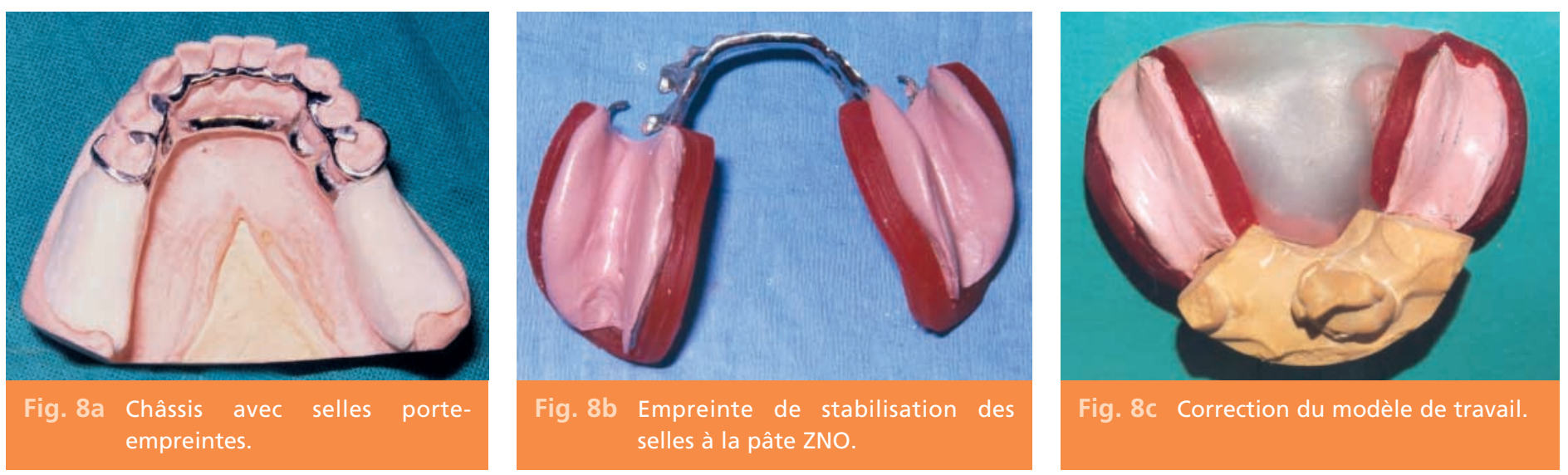

Fig. 8 a à c Empreinte composée partielle. 


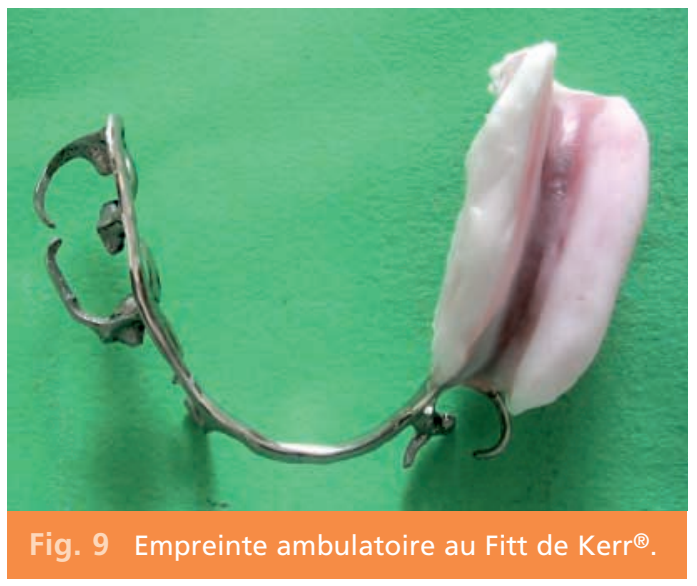

\section{Critères de choix des matériaux et techniques d'empreinte : discussion}

Devant la multiplicité des matériaux de plus en plus «performants» actuellement sur le marché, le praticien doit réagir avec prudence et rigueur évitant ainsi le piège fatal de la simplicité[21].

La compréhension du comportement et des limites de chaque matériau et la connaissance des techniques d'empreinte sont donc indispensables à la conduite avec succès du traitement prothétique.

Cependant, tous les types d'édentements ne sont pas abordés de la même manière.

Chaque cas est analysé et traité différemment, faisant appel à une technique d'empreinte particulière et à des matériaux adaptés, tenant compte de plusieurs facteurs.

En effet, si le regroupement des édentements en édentement à appui dento-parodontal et en édentement à appui mixte a pu simplifier l'approche prothétique de l'édenté partiel en général et le choix des techniques et des maté- riaux d'empreinte en particulier, certaines exigences cliniques et techniques peuvent modifier et orienter le choix différemment :

- Les contres dépouilles au niveau des tissus ostéomuqueux, lorsqu'elles présentent des obstacles par rapport à l'axe d'insertion, peuvent être corrigées chirurgicalement. Mais si leur passage est possible, I'utilisation des matériaux élastiques s'avère nécessaire, les polyéthers répondent mieux à ces exigences cliniques[12].

- Une fibromuqueuse fine, fragile et adhérente au périoste, contre indique toute empreinte compressive comme l'empreinte en deux temps ou "washtechnic».

- L'hypersialorrhée, observée chez les parkinsoniens par exemple, exige des matériaux hydrophiles. L'alginate, les polyéthers, les silicones hydroactifs et la pâte à l'oxyde de zinc sont les matériaux de choix pour une salive abondante. Les polysulfures par leur 
caractère hydrophobe ne peuvent être indiqués.

- L'hyposialorhée ou sécheresse buccale rencontrée chez les diabétiques, les irradiés et les fumeurs contre indique la pâte à l'oxyde de zinc qui peut provoquer des lésions de type brûlures. De plus, ce matériau est très difficile à décoller des muqueuses sèches et fragiles[7].

- L'étendue de la surface d'appui maxillaire surtout en présence de palais profond, impose l'utilisation des matériaux non compressifs, s'évacuant rapidement du porteempreinte. La pâte à l'oxyde de zinc (matériau de prédilection en Prothèse Amovible), assez compressif au moment de sa spatulation, est en réalité à classer parmi les matériaux non compressifs. Les polysulfures de moyenne viscosité sont compressifs sauf pour des palais plats à muqueuse adhérente au périoste ou pour la mandibule où la surface d'appui est réduite[7].

- L'enregistrement des détails (fraisages) des éléments fixés répartis sur l'arcade, fait appel à des matériaux de haute précision comme les silicones et les polyéthers. Ces derniers, du fait de leur viscosité assez faible à l'insertion, puis très haute après la prise répondent mieux à ces exigences. Les études montrent que les polyéthers sont plus adaptés à la réalisation d'empreintes monophasées avec un matériau en une seule viscosité, alors que les polyvinylsiloxanes semblent mieux convenir pour les empreintes en double mélange[12].

- Les cas de résorption importante nécessitent l'utilisation de matériaux compressifs; les polyéthers répondent mieux à ces exigences[23, 24].

- La mobilisation d'un voile en position haute nécessite un matériau avec un temps de prise relativement long. Les silicones ne peuvent être utilisés[7].

- Les sujets âgés, nerveux, anxieux, hypertendus, parkinsoniens, épileptiques ou présentant des réflexes nauséeux, nécessitent une technique d'empreinte simple et rapide enregistrant la totalité des surfaces d'appui quelque soit le type, l'étendue et la situation de l'édentement. Les alginates et les silicones par leur temps de prise relativement court s'adaptent mieux à ces situations. Les polysulfures par leur temps de prise long associé à leur goût désagréable, ne peuvent être indiqués[22].

- Le traitement de l'empreinte, quand il ne peut pas s'effectuer immédiatement en raison de l'éloignement du laboratoire de prothèse par exemple, le choix s'oriente vers les polyéthers, les polyvinylsiloxanes ou la pâte à l'oxyde de zinc.

- L'absence de l'assistante au fauteuil limite le choix des techniques d'empreinte. L'empreinte en un seul temps et avec un matériau en double viscosité (technique du double mélange) devient difficile voir impossible. L'apparition actuelle des pistolets automélangeurs des silicones et des polyéthers a pu élargir ce choix.

- Enfin, le type de la prothèse n'est pas sans incidence sur le choix des techniques et des matériaux d'empreinte. Ainsi, pour la réalisation d'une prothèse provisoire, une empreinte à l'alginate associée à un porteempreinte de commerce suffit largement. 


\section{Conclusion}

L'enregistrement des surfaces d'appui de l'édenté partiel ne fait pas appel à une technique d'empreinte unique ou à un matériau miracle, mais demande une réflexion, une maîtrise des techniques d'empreinte et une rigueur dans leur mise en œuvre.

La connaissance des propriétés fondamentales des matériaux à empreinte et des paramètres multiples et variés ayant une influence sur la valeur de l'empreinte est indispensable.

Selon Bois et coll, une des étapes de la «chaîne» prothétique schématisée par la formule «2 P 2 E 2 R» (Préparer, Protéger, Enregistrer, Exploiter, Reproduire, Respecter) doit être réalisée pour optimiser les chances de réussite[21].

\section{Bibliographie}

1. Batarec E, Buch D.

Abrégé de Prothèse

Adjointe Partielle.

Paris : Editions Masson 1989.

2. Augereau $D$, Renault $P$,

Pierrinard $\mathrm{L}$, loir $\mathrm{F}$,

Fornaro C.

Contraintes

et déplacements dentaires

en Prothèse Adjointe

Partielle :

Analyse par la méthode

des éléments finis.

Cah de Proth 1997;98:88-99.

3. Borel JC, Schittly J,

Exbrayat J.

Manuel de Prothèse

Adjointe Partielle.

Paris : Editions Masson 1994

4. Goumy R, Daulce Goumy V. Techniques d'empreintes en Prothèse Adjointe.

Paris : Editions Masson 1987.

5. Gibert $Y$, Dirat C, Blandir M. L'empreinte secondaire en Prothèse Adjointe Complète :

Principes - technique. Information dentaire 1979;61(15):1261-1268.
6. Naser B, Postaire M. Élastomères et joint périphérique en Prothèse Complète maxillaire. Cah de Proth 1991;75:47-56.

7. Pompignoli M, Doukhan JY, Raux D. Prothèse Complète. Tome 1. Paris : Editions CDP, 1993.

8. Schoendorff $R$, Millet $C$. Rétention en Prothèse Complète. Encycl Méd Chir Odontologie 1995; 23.325.B.05:1-6.

9. Serre $D$, Pouysségur V. Matériaux à empreinte. Encycl Méd Chir Odontologie 1998; 23.064.A.10:1-13.

10. Degrange $M$. Structure, propriétés des élastomères et précision des empreintes. Actual - Odontostomatol 1995; 191:369-385.

11. Degrange $M$, Caire $T$, Eid N.
Caractéristiques générales

et variations

dimensionnelles

des élastomères.

J Biomater dent 1986;2:

73-83.

12. Hoornaert A, Chalard F

Unger $\mathrm{J}$.

Les empreintes aux polyéthers.

Cah de Proth 1997;98:73-84.

13. Bastini $A$,

Fleiter B, Degrange M.

Étude clinique, physique et mécanique

de nouveaux matériaux

à empreinte élastiques. Inf dent 1989;38:3625-3633.

14. Chavaux E, Verdino J,

Lin $M$, Cerisier $P$.

La mouillabilité des silicones hydrophiles. Cah de Proth 1995;91:36-41.

15. Aymé JC, Emery J.

L'adhésion, élément de rétention en Prothèse Adjointe : Étude expérimentale de l'énergie de surface de la résine. 
Cah de Proth 1989;68: 111-118.

16. Lecerf J, Merlet $Y$, Jaijarat $\mathrm{P}$, Borel $\mathrm{M}$. Précision comparée des élastomères, alginates, pâtes thermoplastiques, hydocolloïdes réversibles en Prothèse scellée. Quest Odonto stomatol 1979;14:55 - 69.

17. Anderson JN. The dimensional stability of three silicone base impression materials. Dent Pract and Dental Record 1958;8:368-372.

18. Millet $C$, Allard $Y$ Empreintes secondaires en Prothèse Complète : utilisation

d'un nouveau silicone. Clinic 2003;24(10):667-672.

19. Decup F, Hutin De Swardt I, Renault $\mathrm{P}$, Begin $\mathrm{M}$. Les empreintes en Prothèse Amovible Partielle. Réalités cliniques 1995;6(4):431-445.

20. Duplantier D, Moulin P. Optimisation des empreintes dans les cas d'édentements postérieurs mandibulaires. Cah de Proth 2005;13:35-40.

21. Bois $D$, Allard $Y$, Malquarti G.

Les propriétés fondamentales des produits d'empreintes. Réal Odontostomatol 1990;9(3):181-186.

22. Begin P, Dupas PH. Pratique clinique des matériaux dentaires en Prothèse Fixée : guide clinique. Paris : Editions CDP, 1997.

23. Taib.M. Le joint palatin postérieur son importance, sa conception.

Cah de Proth 1976;13: 103-112.

24. Begin $\mathrm{M}$, Rohr M. Le joint vélo-palatin en Prothèse Complète Maxillaire.

Cah de Proth 1983;43:55-78.

\section{SUMMARY}

\section{Selection criteria of materials}

and impression's techniques in partial removable prosthesis

Nadia MERZOUK

Salwa BERRADA

Faiza BENFDIL

Ahmed ABDEDINE

$$
\begin{aligned}
& \text { Keywords } \\
& \text { - tissue duality } \\
& \text { - peripheral joint } \\
& \text { - impression's materials } \\
& \text { - impression's techniques } \\
& \text { - selection criteria }
\end{aligned}
$$

The impression constitutes a capital stage of the realization of a partial removable prosthesis.

It takes part in the research of prosthetic balance and must hold account the various physiological behaviours of the support's structures.

After the study of the principal properties of impression's materials and a short description of the impression's techniques, we propose an analysis of the possible clinical factors and techniques having an incidence on the choice of impression's materials and impression's techniques. 


\section{Questions - Réponses}

1 - Sous une même pression occlusale, la dépressibilité de la fribromuqueuse saine est ? a - La même que celle du desmodonte

b - Plus importante que celle du desmodonte

c - Moins importante que celle du desmodonte

q : əsuodəoy

2 - Quand faut- dissocier l'enregistrement des surfaces d'appui, ostéo-muqueuse et dentoparodontale?

a - dans la cl IV de Kennedy Applegate (KA)

b - dans la cl III de KA

c - dans la cl I de KA

d - dans la cl II de KA

$p$ 'o 'e : əsuodọy

3 - Chez le sujet irradié, la matériau d'empreinte à éviter est :

a - le polysulfure

b - le silicone

c - le polyether

$\mathrm{d}$ - la pâte à l'oxyde de zinc

$p$ : əsuodòy 